\title{
Budget impact model of avelumab in patients with metastatic merkel cell carcinoma in the US
}

This article was published in the following Dove Press journal:

ClinicoEconomics and Outcomes Research

\author{
Murtuza Bharmal' \\ Mairead Kearney' \\ Ying Zheng ${ }^{2}$ \\ Hemant Phatak ${ }^{2}$ \\ 'Global Evidence and Value \\ Development, Merck Healthcare KGaA, \\ Darmstadt, Germany; ${ }^{2}$ US Health \\ Economics and Outcomes Research, \\ EMD Serono, Inc, Rockland, MA, USA
}

Correspondence: Murtuza Bharmal Global Evidence and Value Development, Merck Healthcare KGaA, 250 Frankfurter Street, Darmstadt, Germany

Email

murtuza.bharmal@merckgroup.com

\begin{abstract}
Objective: To estimate the budget impact of avelumab as a treatment option for patients with treatment-naïve first-line (1L) and previously treated second-line or later $(2 \mathrm{~L}+)$ metastatic Merkel cell carcinoma (mMCC) in the US.

Methods: A budget impact model was developed to evaluate the addition of avelumab for the treatment of mMCC patients using a hypothetical 30 million-member US health plan over a 3-year time horizon (2019-2021). The comparator treatments included in the analysis were pembrolizumab and nivolumab (other immuno-oncology agents); and the chemotherapies routinely used in the eligible mMCC population. Model inputs included market share uptake of avelumab and other comparators, duration of treatments, and costs (drugs, health care resource utilization, adverse events). The model was evaluated from a commercial payer perspective. Sensitivity analyses were conducted to test uncertainties arising from the input values used in the model.
\end{abstract}

Results: In a hypothetical commercial health plan of 30 million members, 285 patients with mMCC were identified over 3 years; 43 patients received avelumab as a 1L treatment over 3 years. In a world without avelumab, the total health care costs of treating patients with mMCC over 3 years were estimated to be US\$11,710,115 from a commercial health plan perspective. With avelumab, there were estimated savings of $\$ 2,643,173$ considering the total costs related to the treatment of mMCC over 3 years (23\% reduction in the budget). The incremental cost per member per month over 3 years was $-\$ 0.0025$.

Conclusion: The model results indicate that the adoption of avelumab as a treatment option for mMCC would likely result in minimal budget impact from a US health plan perspective. Patients with $\mathrm{mMCC}$, a rare condition with a poor prognosis and high unmet need, may benefit greatly from recently approved immunotherapies.

Keywords: metastatic merkel cell carcinoma, cost, budget impact model, avelumab

\section{Introduction}

Merkel cell carcinoma (MCC) is a rare cutaneous neuroendocrine malignancy. The etiology of MCC is likely multifactorial, with risk factors including immunosuppression, autoimmune conditions, previous Merkel cell polyomavirusinfection, advancing age, fair skin, and ultraviolet light exposure. ${ }^{1-3}$ Between 2000 and 2013, the number of reported solid tumor cancer cases $(6,600$ cases identified by the SEER-18 database) increased by $15 \%$, melanoma cases increased by $57 \%$, and MCC cases increased by $95 \% .{ }^{4}$ In 2013, the MCC incidence rate was 0.7 cases per 100,000 person-years in the US, corresponding to 2,488 cases per year. ${ }^{4}$ Other factors, such as older age, male gender, and Caucasian ethnicity, have been shown to be associated with the increased incidence of MCC in the US. ${ }^{4-6}$ For example, the incidence of MCC increased from 0.1 to 1.0 to 9.8 (per 100,000 person-years) among age groups 
40-44 years, 60-64 years, and $\geq 85$ years, respectively; and the US MCC incident cases are expected to climb to 2,835 cases per year in 2020 and 3,284 cases per year in 2025 due to the aging population. ${ }^{4}$

Approximately $4-15 \%$ of MCC patients are diagnosed at the metastatic stage; the propensity for metastasis is high, occurring in over $30 \%$ of cases within $1-3$ years following diagnosis. ${ }^{7-9}$ Survival is strongly dependent on disease stage at diagnosis, with a worse prognosis observed with advanced and metastatic MCC (mMCC).${ }^{10}$ In patients with mMCC, the 5-year survival rates fall considerably, with estimates ranging from $0 \%$ to $18 \%{ }^{11,12}$ Studies have shown that the median survival from the time of initial metastasis was approximately 9.6 months in MCC patients who developed distant metastatic disease. ${ }^{8}$ Within the US, MCC patients who had distant $\mathrm{mMCC}$ and who were undergoing second-line or later $(2 \mathrm{~L}+)$ chemotherapy, demonstrated very low survival. ${ }^{13,14}$ In the most recent US study conducted using US Oncology Network data, a total of 20 patients who qualified for $2 \mathrm{~L}+$ therapy and previously treated with first-line (1L), had a 6-month overall survival (OS) rate of $30.2 \%$ (95\% CI: $11.6-51.4)$ and a 12 -month OS rate of $0.0 \%{ }^{14}$

Avelumab, an anti-PD-L1 antibody, was approved by the FDA in March 2017, as the first anti-PD-L1 immunotherapy for adult and pediatric patients. In December 2018 , the FDA granted accelerated approval to pembrolizumab for adult and pediatric patients with recurrent locally advanced or mMCC. Although not yet approved for the treatment of mMCC, nivolumab has been recommended for use in NCCN guidelines. ${ }^{15}$ Prior to the approval of avelumab, treatment options have been palliative and included: participation in clinical trials, chemotherapy as clinical judgment dictated for patients with contraindications to checkpoint immunotherapy, and radiation therapy. ${ }^{15,16}$ Systemic chemotherapy has been commonly used as a palliative option and although studies report initial responses to chemotherapy, the duration of response is short and the true impact on survival remains unclear. ${ }^{15,17-22}$ Responses are lower in the second-line (2L) setting (23\%) compared with the 1L (53-57\%). ${ }^{13,14,17,22}$ Additionally, the chemotherapy options commonly used are associated with considerable toxicity, rendering them unsuitable for use in many mMCC patients who tend to be elderly, with poor Eastern Cooperative Oncology Group (ECOG) performance status, and with multiple comorbidities. ${ }^{22-25}$
It is important to evaluate the financial impact that a new therapy approved for patients with mMCC may have on the current US healthcare system. Budget impact models (BIMs) are used by payers and other health care decision-makers to estimate the incremental expenditures of adopting a new treatment to drug formularies on a health plan. The primary objective of this study is to evaluate the budget impact from a US health plan payer perspective of the introduction of avelumab for the treatment of mMCC patients who are treatment-naïve (1L) and previously treated $(2 \mathrm{~L}+)$.

\section{Methods}

\section{Model structure, perspective, and time horizon}

An economic model was developed in Microsoft ${ }^{\circledR}$ Excel ${ }^{\circledR}$ (v16.0, 32-bit) to assess the budget impact of avelumab for the treatment of mMCC patients in a hypothetical 30 million-member $(30,000,000)$ US health plan over a 3-year time horizon. Model cycle length was set at 4 weeks. The BIM allocated eligible patients to the various treatment options and compared the projected uptake of avelumab (world with avelumab, revised scenario) with various alternative treatment options (world without avelumab, current scenario) (Figure 1). The BIM contained information on the number of eligible patients for treatment of $\mathrm{mMCC}$, the market shares of current and anticipated treatment distributions for all of the interventions compared, and the relevant drug and health care resource use (HRU) costs. The costs were calculated based on the number of eligible patients, treatment duration, adverse events (AEs), and market share of various treatments. The budget impact was the difference between the total costs from the "world without avelumab" scenario and the "world with avelumab" revised scenario. The model was evaluated using a commercial payer perspective. No discounting was required because the aim of the BIM was only to compare annual nominal cash flows. The BIM was set to a 3-year time horizon (2019-2021).

\section{Model input variables} Target population

The BIM estimated the population within a 30 million-member health plan who were identified with MCC. The final patient population for the model included $1 \mathrm{~L}$ and $2 \mathrm{~L}+$ patients diagnosed with mMCC. In 2019, 252 patients were estimated to have MCC, within a population of 30 million, and $37 \%$ (94 patients) were assumed to have mMCC with 25 patients eligible for 1L treatment, and 7 patients eligible for 


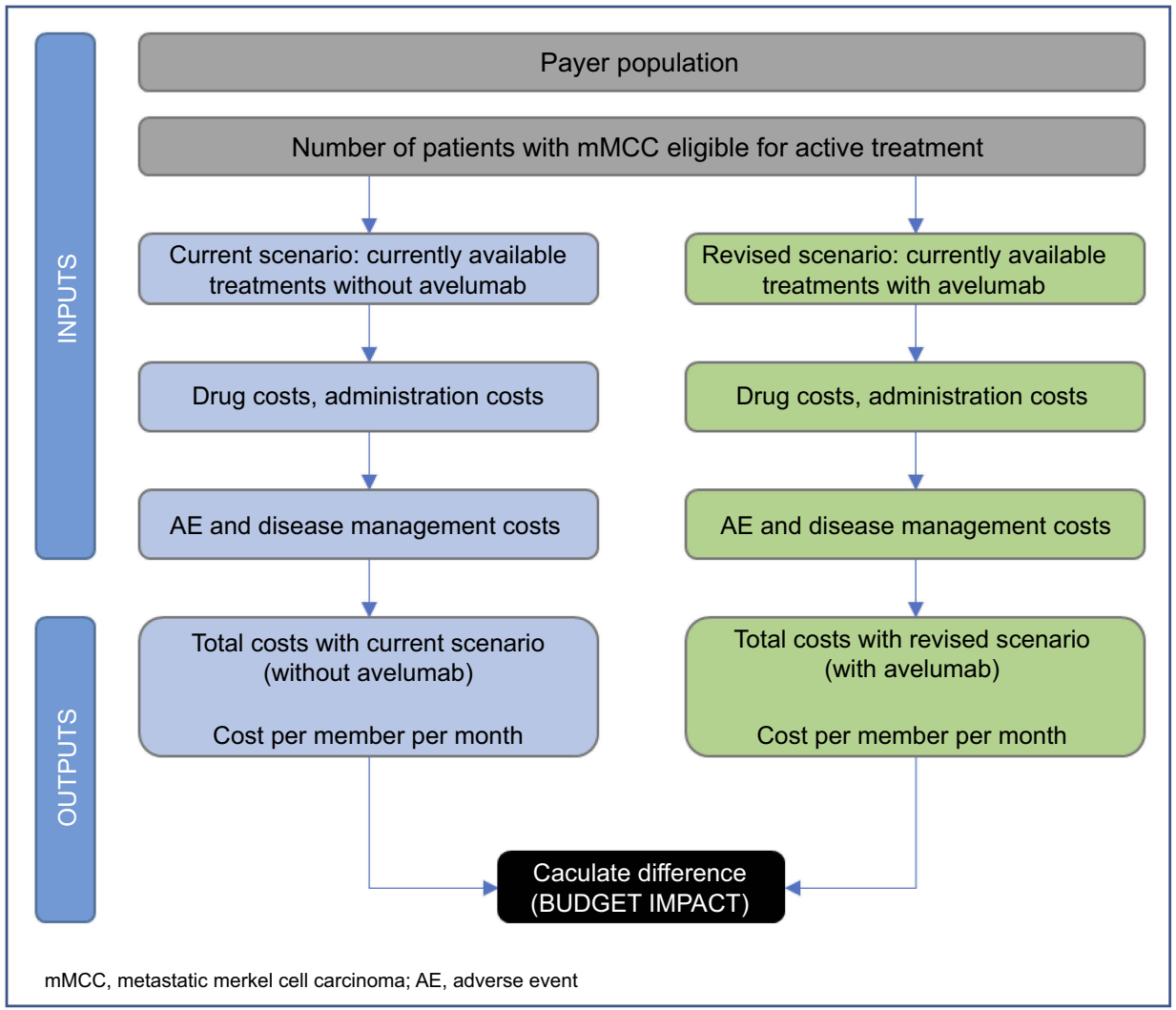

Figure I Model structure.

2L+ (Table 1). From the years 2019 to 2021, a total of 97 patients were estimated to be eligible for treatment. The disease incidence rate was identified in published literature. ${ }^{4}$

\section{Treatments and market share}

The comparator treatments in $1 \mathrm{~L}$ and $2 \mathrm{~L}+$ included other immuno-oncology (IO) agents (pembrolizumab and nivolumab) and the chemotherapies routinely used in the eligible population. It was assumed that IO agents would partially replace chemotherapies. Within the model, the estimated market share of IO agents was set at $80 \%$ in the $1 \mathrm{~L}$ setting and $25 \%$ in $2 \mathrm{~L}+$, with the remaining $20 \%$ and $75 \%$ of $\mathrm{mMCC}$ patients being treated with chemotherapies in $1 \mathrm{~L}$ and $2 \mathrm{~L}+$, respectively (Table 2). Within the IO agents, avelumab was assumed to have a consistent uptake at $65 \%$ each year for 3 years, pembrolizumab at $28 \%$, and nivolumab at $7 \%$. The market share of chemotherapies was set at the same rate for the 3 years at $20 \%$ as $1 \mathrm{~L}$ and $75 \%$ as $2 \mathrm{~L}+$ (Table 2 ). A total of $44 \%$ of patients treated with chemotherapies received topotecan with the remainder receiving a range of alternative chemotherapies. The market share assumptions were based on an internal forecast (Merck Healthcare KGaA/EMD Serono, Inc., data on file) and a study by Cowey et al 2017 (Table 2). ${ }^{14,}$

\section{Cost estimation}

This model included the following types of costs in the analysis: drug costs, HRU/management costs and AE (grade 3+) costs. Chemotherapy costs were calculated using bodyweight-based dosing. The costs of IO agents (avelumab, pembrolizumab, and nivolumab) and carboplatin were based on

Table I Patient population in 2019

\begin{tabular}{|l|l|l|l|}
\hline Data description & Rate & Value & Source \\
\hline Plan population & NA & $30,000,000$ & User Input \\
MCC incidence rate (per 100,000) & 0.84 & 252 & 4,52 \\
mMCC (\%) & $37 \%$ & 94 & Calculated from Cowey et al, 2017 ${ }^{14}$ \\
Total number of patients receiving treatment (includes IL and 2L+) & $34 \%$ & 32 & Calculated from Cowey et al, 2017 \\
\hline
\end{tabular}

Abbreviations: MCC, merkel cell carcinoma; mMCC, metastatic merkel cell carcinoma; IL, treatment-naïve, first line; $2 \mathrm{~L}+$, second-line or later. 
Table 2 Market shares of therapies in the $I L$ and $2 L+$ settings

\begin{tabular}{|c|c|c|c|}
\hline & $\begin{array}{l}2019-2021 \text {, without } \\
\text { avelumab }\end{array}$ & $\begin{array}{l}2019-2021 \text {, with } \\
\text { avelumab }\end{array}$ & Source \\
\hline \multicolumn{4}{|l|}{ IL } \\
\hline IO Agents & $80.0 \%$ & $80.0 \%$ & \multirow[t]{4}{*}{ Merck Healthcare KGaA/EMD Serono, Inc., data on file } \\
\hline Avelumab & $0.0 \%$ & $65.0 \%$ & \\
\hline Pembrolizumab & $80.0 \%$ & $28.0 \%$ & \\
\hline Nivolumab & $20.0 \%$ & $7.0 \%$ & \\
\hline Chemotherapy & $20.0 \%$ & $20.0 \%$ & \multirow[t]{5}{*}{ 14, Merck Healthcare KGaA/EMD Serono, Inc., data on file } \\
\hline Carboplatin & $11.1 \%$ & $11.1 \%$ & \\
\hline $\begin{array}{l}\text { Carboplatin+ } \\
\text { etoposide }\end{array}$ & $11.1 \%$ & $11.1 \%$ & \\
\hline $\begin{array}{l}\text { Cyclophosphamide+ } \\
\text { doxorubicin+ } \\
\text { vincristine }\end{array}$ & $33.3 \%$ & $33.3 \%$ & \\
\hline Topotecan & $44.4 \%$ & $44.4 \%$ & \\
\hline \multicolumn{4}{|l|}{$2 \mathrm{~L}+$} \\
\hline IO Agents & $25.0 \%$ & $25.0 \%$ & \multirow[t]{4}{*}{ Merck Healthcare KGaA/EMD Serono, Inc., data on file } \\
\hline Avelumab & $0.0 \%$ & $65.0 \%$ & \\
\hline Pembrolizumab & $80.0 \%$ & $28.0 \%$ & \\
\hline Nivolumab & $20.0 \%$ & $7.0 \%$ & \\
\hline Chemotherapy & $75.0 \%$ & $75.0 \%$ & \multirow[t]{5}{*}{ I4, Merck Healthcare KGaA/EMD Serono, Inc., data on file } \\
\hline Carboplatin & $11.1 \%$ & $11.1 \%$ & \\
\hline $\begin{array}{l}\text { Carboplatin + } \\
\text { etoposide }\end{array}$ & $11.1 \%$ & $11.1 \%$ & \\
\hline $\begin{array}{l}\text { Cyclophosphamide + } \\
\text { doxorubicin }+ \\
\text { vincristine }\end{array}$ & $33.3 \%$ & $33.3 \%$ & \\
\hline Topotecan & $44.4 \%$ & $44.4 \%$ & \\
\hline
\end{tabular}

Abbreviations: IL, treatment-naïve, first line; $2 \mathrm{~L}+$, second-line or later; IO, immuno-oncology.

flat doses. ${ }^{26-28}$ No discounting of costs was applied to the BIM, as per established by BIM guidelines from the International Society of Pharmacoeconomics and Outcomes Researchand formulary submission guidelines from the Academy of Managed Care Pharmacy. ${ }^{29,30}$ All costs obtained before 2018 were inflated to 2018 using the medical care component of the Consumer Price Index from the United States Department of Labor. ${ }^{31}$

Treatment duration and costs. No differentiation of treatment duration by response was applied in the $1 \mathrm{~L}$ setting given a lack of data. Additionally, as median treatment duration was not reported in the most recently published trial data for IO agents, median progression-free survival (PFS) was used as a proxy. ${ }^{32,33}$ Treatment duration for nivolumab was assumed to be the same as pembrolizumab and was based on the median PFS observed in clinical trials. ${ }^{33}$ For $1 \mathrm{~L}$ treatment, duration of chemotherapy was also based on clinical trial results. ${ }^{14}$ The duration of response was varied for different treatments and separately by responders and non-responders in the sensitivity analysis to test the robustness of the assumptions. Within the $2 \mathrm{~L}$ + setting, the treatment duration of IO agents was assumed to vary for responders and non-responders based on prior evidence and estimates used in a cost-effectiveness model 
Table 3 Duration of treatment (months)

\begin{tabular}{|l|l|l|l|}
\hline & $\begin{array}{l}\text { Responders } \\
\text { (CR+PR) }\end{array}$ & $\begin{array}{l}\text { Non-responders } \\
\text { (SD+PD) }\end{array}$ & Source \\
\hline IL & & & \\
Avelumab & 9.1 & 9.1 & 32 \\
Pembrolizumab & 16.8 & 16.8 & 33 \\
Nivolumab & 16.8 & 16.8 & 33 , same as pembrolizumab (assumed) \\
Chemotherapies & 4.6 & 4.6 & 14 \\
\hline 2L+ & & & Responders: 35; Non-responders: 34 \\
Avelumab & 4.4 & 2.7 & Responders: 35; Non-responders: 34, same as avelumab (assumed) \\
Pembrolizumab & 4.4 & 2.7 & Responders: 35; Non-responders: 34, same as avelumab (assumed) \\
Nivolumab & 4.4 & 2.7 & 14 \\
Chemotherapies & 2.2 & 2.2 & \\
\hline
\end{tabular}

Abbreviations: IL, treatment-naïve, first line; $2 \mathrm{~L}+$, second-line or later; $C R$, complete response; PR, partial response; SD, stable disease; PD, progressive disease.

Table 4 Summary of costs (per 4-week model cycle)

\begin{tabular}{|c|c|c|c|}
\hline Treatments & $\begin{array}{l}\text { Drug } \\
\text { costs } \\
\text { (USD) }\end{array}$ & $\begin{array}{l}\text { HRU } \\
\text { costs } \\
\text { (USD) }\end{array}$ & $\begin{array}{l}\text { AE } \\
\text { costs } \\
\text { (USD) }\end{array}$ \\
\hline \multicolumn{4}{|l|}{10 Agents } \\
\hline Avelumab & 12,894 & 696 & 375 \\
\hline Pembrolizumab & 12,953 & 464 & 67 \\
\hline Nivolumab & 13,034 & 348 & 67 \\
\hline \multicolumn{4}{|l|}{ Chemotherapy } \\
\hline Carboplatin & 39 & 331 & 433 \\
\hline Carboplatin+etoposide & 152 & 766 & $\mathrm{I}, 334$ \\
\hline $\begin{array}{l}\text { Cyclophosphamide+doxorubi- } \\
\text { cin+vincristine }\end{array}$ & 1,173 & 442 & 886 \\
\hline Topotecan & 207 & 1,214 & 3,287 \\
\hline
\end{tabular}

Abbreviations: $\mathrm{HRU}$, health care resource utilization; IO, immuno-oncology; $A E$, adverse event.

based on trial data (Table 3). ${ }^{34,35}$ For $2 \mathrm{~L}+$ chemotherapy, duration of treatment was based on clinical study results. ${ }^{14}$ The duration of treatment was assumed to be similar across all the chemotherapy regimens. Unit costs of drugs were obtained from the CMS drug pricing files (accessed in 2018). ${ }^{36}$ All the treatments considered in the analysis were intravenous, and their respective administration costs were included in the analysis. The drug costs are outlined in Table 4.

$A E$ Costs. Based on the National Cancer Institute Common Terminology Criteria for Adverse Events, the analysis included grade 3 or higher AEs occurring in more than $5 \%$ of patients with at least one treatment. AEs considered in the analysis included neutropenia, alanine transaminase/increased transaminase, anemia, leukopenia/lymphopenia, nausea/vomiting, decreased hemoglobin, fatigue, dyspnea, infections, hyponatremia, elevated aspartate aminotransferase, myelosuppression, neuropathy, and thrombocytopenia. The rates of AEs were obtained from clinical trials and treatment labels. ${ }^{26-28,34,37-41}$ The proportion of severe AEs to non-severe AEs was derived from the literature and used as a proxy to determine the ratio of hospitalizations and outpatient visits for each $\mathrm{AE} .{ }^{42}$ The cost of each AE was extracted using CPT codes for outpatient costs and HCUPnet data for hospitalizations. ${ }^{43-}$ 45 After applying a weighted average to the costs (weighted for ratio of hospitalizations and outpatient visits), the three most expensive AEs were thrombocytopenia, dyspnea, and neutropenia, at US\$3,142, $\$ 2,058$, and $\$ 1,715$, respectively. For avelumab, other IO agents and various chemotherapies the frequency of thrombocytopenia was $1.0,0.0$, and $0.0-50.2 \%$, for dyspnea was $1.0,0.0$, and $0.0-6.1 \%$, and for neutropenia was $1.0,0.0$, and $0.0-49.8 \%$, respectively. The total $\mathrm{AE}$ costs are displayed in Table 4.

Healthcare resource utilization. HRU costs associated with mMCC treatments included intravenous administration (first and subsequent hours), hospitalization, general physician and specialist visits, and some tests and scans. Frequencies of HRU were provided by ECOG reports, clinical trials, and other literature sources. ${ }^{37,41,46,47}$ Unit costs associated to monitoring, physician visits, scans, and tests were sourced from the fee schedules, and they applied for the duration of treatment. ${ }^{48,49}$ The HRU costs are displayed in Table 4.

\section{Results}

In a world without avelumab, using a base-case analysis of a $30,000,000$ member-health plan, the total cost for treating patients with mMCC over 3 years (2019-2021) was estimated to be $\$ 11,710,115$ from a payer perspective. The total savings associated with the introduction of avelumab 
Table 5 Budget impact over 3 years, 2019-202I (USD)

\begin{tabular}{|l|l|l|l|l|}
\hline Costs & $\begin{array}{l}\text { Scenario without } \\
\text { avelumab } \\
\text { (A) }\end{array}$ & $\begin{array}{l}\text { Scenario with } \\
\text { avelumab } \\
\text { (B) }\end{array}$ & $\begin{array}{l}\text { Cumulative budget } \\
\text { impact } \\
\text { (C = B - A) }\end{array}$ & $\begin{array}{l}\text { \% change in } \\
\text { budget }\end{array}$ \\
\hline Drugs & $\$ 11,178,202$ & $\$ 8,523,446$ & $-\$ 2,654,756$ & $-23.8 \%$ \\
HRU & $\$ 478,837$ & $\$ 479,957$ & $0.2 \%$ \\
AEs & $\$ 53,076$ & $\$ 63,540$ & 1,120 & $10.7 \%$ \\
Total & $\$ 11,710,115$ & $\$ 9,066,942$ & $-\$ 2,643,173$ & $22.6 \%$ \\
Cost per member per month & $\$ 0.0109$ & $\$ 0.0084$ & $-\$ 0.0025$ & $\mathbf{2 2 . 6 \%}$ \\
\hline
\end{tabular}

Note: Bold values indicate key conclusions of the analysis: total and per member per month costs.

Abbreviations: $\mathrm{HRU}$, health care resource utilization; $A E$, adverse event.

over 3 years were $\$ 2,643,173$, driven mainly by the decrease in drug costs over the 3-year period ( $23 \%$ reduction in the budget) (Table 5). The incremental cost per member per month (PMPM) was $-\$ 0.00212$ in the first year, $-\$ 0.00260$ in the second year, and $-\$ 0.00262$ in the third year.

\section{Sensitivity analyses (SA)}

Deterministic one-way SA were performed to examine the uncertainty around key parameters on model outputs. Treatment duration was subject to much uncertainty, and several different scenarios were tested to address various possibilities. One example of uncertainty included the median PFS of pembrolizumab, a proxy for treatment duration, with trial data reported as 16.8 months $(95 \%$ CI, 4.6 months to not estimable). ${ }^{33}$ As the $95 \%$ confidence interval was wide, two scenarios tested the approximate limits of the interval, reducing treatment duration to a quarter and increasing it by $300 \%$ for pembrolizumab and nivolumab. These variations resulted in a 3-year incremental budget impact of $\$ 7,961,329$ and $\$ 2,146,493$, respectively, demonstrating a wide range of potential impacts. Additionally, the pembrolizumab trial reported a median PFS longer than the avelumab trial, driving a large difference in treatment duration between IO agents in this analysis. ${ }^{32,33}$ Two scenarios were tested to address uncertainty regarding this difference. One scenario assumed the $1 \mathrm{~L}$ duration of all IO agents was based on the avelumab trial data and the other assumed $1 \mathrm{~L}$ duration was based on the pembrolizumab trial data and the 3-year budget impact was $\$ 74,306$ and $\$ 110,810$, respectively, both relatively negligible increases compared to the overall budget. Additionally, as the duration of $1 \mathrm{~L}$ non-responders was assumed to be the same as responders (for both IO agents and chemotherapies) due to the lack of data, one scenario tested a $50 \%$ reduction in $1 \mathrm{~L}$ treatment duration for nonresponders and found an incremental budget impact of $\$ 2,099,499$ over 3 years. Duration of treatment for all IO agents in the $1 \mathrm{~L}$ was also doubled and halved and these scenarios were found to have incremental budget impacts of $-\$ 3,630,874$ and $-\$ 1,612,051$, respectively.

Market shares scenario included a slower uptake of IO agents in $1 \mathrm{~L}$, where their market share was $50 \%$ in $1 \mathrm{~L}$ and increased to $50 \%$ in $2 \mathrm{~L}+$ (exclusive scenario with altered market shares), resulting in a 3-year impact of $-\$ 2,000,186$. Another scenario assumed that IO agents completely replaced chemotherapies in both $1 \mathrm{~L}$ and $2 \mathrm{~L}+$ setting, with an impact of $-\$ 3,060,105$ over 3years. Additional scenarios modifying the incidence of $\mathrm{mMCC}$, number of patients receiving treatment, and treatment duration were all tested separately. More information regarding each scenario and the resulting effect on the incremental budget impact and incremental PMPM cost is presented in Table 6. All the analyses suggested that avelumab has a minimal budget impact, with PMPM consistently below $\$ 0.01$. The largest effect was observed for the scenario which varied treatment duration for $1 \mathrm{~L}$ pembrolizumab and nivolumab.

\section{Discussion}

Survival is poor in patients with $\mathrm{mMCC}$ and early diagnosis and timely interventions are key to improving health outcomes. Prior to the approval of avelumab in 2017, treatment options for mMCC were limited; with most patients being offered chemotherapy or best supportive care. Patient outcomes and survival benefit have been minimal with the use of chemotherapy. Now that avelumab and pembrolizumab have been approved for the treatment of mMCC in the US, the spectrum of current therapies for patients with $\mathrm{mMCC}$ is changing. Several clinical trials of immune checkpoint inhibitors (anti-PD-1, PD-L1, and CTLA-4 Abs) administered as monotherapy or in combination with other agents or modalities are ongoing as a search on ClinicalTrials.gov 


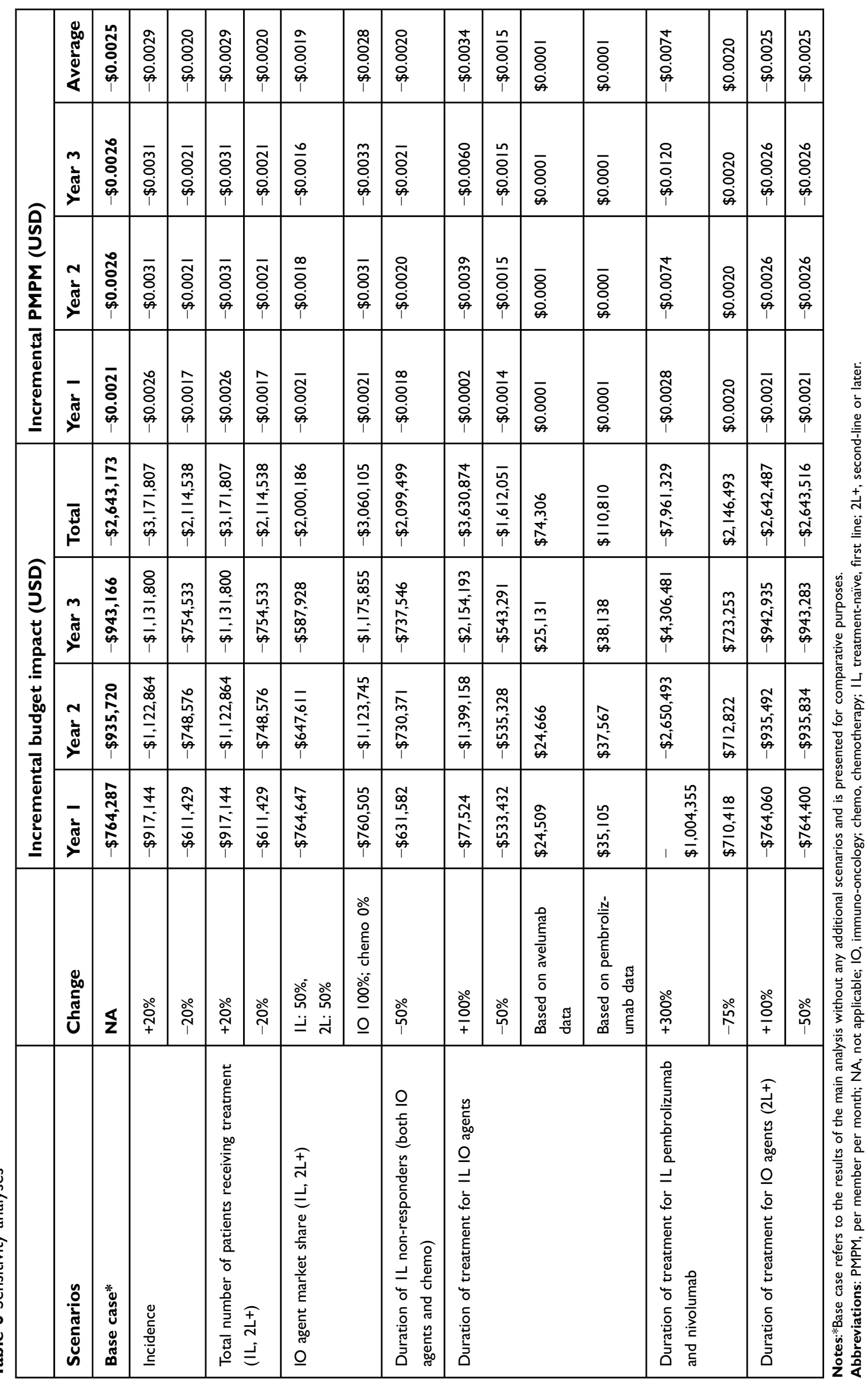


revealed, and these may provide further treatment options for patients with mMCC. With the understanding that mMCC is a rare condition with a poor prognosis, patients have the opportunity to benefit greatly from new innovative immunotherapies. An analysis of real-world data from SEER-Medicare in patients aged $\geq 65$ years diagnosed with mMCC from 2006 to 2013 revealed that $83 \%$ of patients received surgery, radiation or chemotherapy as $1 \mathrm{~L}$ treatment. ${ }^{50}$ The cumulative proportion of newly diagnosed mMCC patients surviving at 1 year was $48 \%$, and $16 \%$ at 5 years. $^{50}$

The purpose of this study was to evaluate the budget impact of the introduction of a new IO agent, avelumab, into the US healthcare system from a payer perspective. Based on results from the JAVELIN Merkel 200 Part A study, a multicenter, international, open-label, Phase II clinical trial, the FDA granted an accelerated approval for avelumab for the treatment of adults and pediatric patients 12 years and older with mMCC. Also, in this trial, there was a trend toward a higher response rate which was observed in patients treated with avelumab who had fewer lines of prior treatment, which along with the pembrolizumab data, strongly suggest that immunotherapy targeting the PD-1 pathway should be considered for the treatment in patients with advanced MCC. ${ }^{33}$ Additionally, due to its promising results from its clinical trial, avelumab has also been included as a treatment option in NCCN guidelines for disseminated MCC. ${ }^{15}$

The HRU cost of avelumab is higher relative to other IO agents, in large part, due to the increased frequencies of administration (eg, more frequent intravenous administrations leads to increased costs). As per package inserts, a flat dose of $800 \mathrm{mg}$ for avelumab was used intravenously every 2 weeks, whereas for other IO agents, cycles vary from 3 to 4 weeks (nivolumab was conservatively estimated to be administered at a dose of $480 \mathrm{mg}$ every 4 weeks). ${ }^{26-28}$ Additionally, the frequency of AEs was obtained from the package insert for avelumab. ${ }^{26}$ For other IO agents, as the package inserts contained incomplete AE frequency information for MCC patients, package inserts were used in conjunction with trial publications. ${ }^{27,28,33,52}$ The available evidence reported different frequencies of AEs between avelumab, which had slightly higher rates of neutropenia, anemia, and leukopenia/lymphopenia relative to other IO agents. Despite the minor differences in $\mathrm{AE}$ frequencies, costs related to AEs were much smaller compared to other cost categories, and the discrepancy between IO agents had a relatively negligible effect on the final result.
SA were conducted varying the effect of key parameters in the BIM, with particularly wide variance used in relation to treatment duration which was subject to greater uncertainty. Across all scenarios, avelumab had a minimal budget impact, with health care costs consistently below $\$ 0.01$ PMPM in large part due to the rarity of the disease in relation to the amount of health plan members. The largest variance was observed in relation to scenarios decreasing treatment duration for pembrolizumab and nivolumab by a quarter and increasing them by $300 \%$. The robustness of these assumptions was limited by the availability of data. Since avelumab has been approved for the treatment of mMCC in the US, EU, Japan, and other markets, the spectrum of current therapies for patients with MCC is evolving creating a need to evaluate the economic impact of novel therapies.

A value-based cost-effectiveness analysis of avelumab was conducted from a UK National Health Service perspective. $^{35}$ The analysis included a three state, partitioned-survival model based on the JAVELIN Merkel 200 trial. A mix of chemotherapy and best supportive care was considered as the standard of care and the introduction of avelumab resulted in an incremental cost-effectiveness ratio (ICER) of $£ 39,178$ in treatment-naïve mMCC patients and $£ 35,274$ in treatment-experienced patients. The ICER was calculated to have a $69.3 \%$ and $88.3 \%$ probability of being cost-effective at a threshold of $£ 50,000$ per quality-adjusted life year for treatment-experienced and treatment-naïve patients, respectively. The analysis included UK-specific inputs and other IO agents were not considered as comparators which is a notable difference from this BIM's US assumptions. However, to the authors' knowledge, this is the only cost-effectiveness analysis published for $1 \mathrm{~L}$ treatment of avelumab in mMCC patients. An additional country-specific analysis with an updated list of comparators is warranted to support these findings in a US setting and validate if the reimbursement of avelumab would be an efficient use of scarce health care resources.

\section{Limitations}

As with any BIM, the validity of the results is only as plausible as the inputs and assumptions made within the model. Assumptions made in this BIM are unlikely to be applicable to all health plans or payer types with different population distributions, formulary structures, and costsharing arrangements. With any study, there are some limitations to address, for example, due to the lack of 
clinical evidence on the efficacy of sequencing of IO agents in both $1 \mathrm{~L}$ and $2 \mathrm{~L}+$ setting, market shares of IO agents were assumed to be stable across the 3-year timeframe of the model. The analysis assumed that the introduction of avelumab only altered the treatment mix of the IO therapy shares and not the shares of chemotherapies. Because there are limited data for many of the parameters, much of the parameter uncertainty in the BIM cannot be meaningfully quantified. To address this, various scenario analyses were undertaken by changing selected input parameter values and structural assumptions to produce plausible alternative scenarios. ${ }^{29}$

As a non-probabilistic model was developed, partly due to insufficient data to create a state-based model, patient-to-patient variance was not incorporated in the model structure and is a limitation. Additionally, only one therapy was considered per patient with no costs from prior (for $2 \mathrm{~L}+$ ) or subsequent therapies limiting the scope of costs for all patients. For inputs such as PFS, median values were reported and subsequently utilized in the BIM. Medians are likely to have right-skewed distributions relative to means which directly represent the entire population. As such, costs may be underestimated as some patients could undergo treatment for a much longer duration than the median PFS and due to asymmetry, patients cannot undergo treatment for a much shorter period than the median PFS. Additionally, a large driver of cost difference occurred due to the gap between median PFS of avelumab and pembrolizumab trial data (used as proxies for treatment duration). This gap may be at least be partly attributed to the differences in populations included in the trials. For instance, the pembrolizumab trial included patients with advanced MCC whereas the avelumab trial included patient with mMCC which likely drives difference in median PFS. Due to limited available data, these trials were used to estimate treatment duration and several SA scenarios were employed to vary these assumptions, test their robustness and evaluate the budget impact with different sets of assumptions. Moreover, increased survival in patients treated with IO agents would also potentially increase the costs related to these patients.

\section{Conclusion}

In conclusion, the results of this BIM analysis indicate that providing avelumab for the treatment of mMCC demonstrated a minimal PMPM budget impact to a US health plan. That was in part due to the small number of patients expected to be eligible for treated with avelumab therapy in $\mathrm{mMCC}$, a rare and aggressive condition with poor prognosis. Hence, the addition of avelumab to formularies offers a novel treatment option for patients with only a modest increase in healthcare expenditure.

\section{Acknowledgments}

This study was sponsored by Merck Healthcare KGaA, Darmstadt, Germany, and is part of an alliance between Merck Healthcare KGaA, Darmstadt, Germany and Pfizer Inc., New York, NY, USA. We would like to acknowledge Olga Ovcinnikova, project lead from Mapi at the time of study; Anna Forsythe, project lead from Purple Squirrel Economics, for overall project support; Dimitrios Tomaras and Nazia Merritt, medical writing support team from Purple Squirrel Economics at the time of the study, for their assistance in drafting the manuscript. Parts of this analysis were previously published in a poster presented at ISPOR EU, 2017 (Bharmal M, D'Angelo SP, Phatak H. Budget impact analysis of avelumab in patients with metastatic Merkel cell carcinoma in the US. Value Health. 2017;20(9): abstract A424. [ISPOR EU 2017]. Abstract No. PCN 70. Presented at the ISPOR 20th Annual European Congress. November 48, 2017; Glasgow, Scotland. https://www.valueinhealthjour nal.com/article/S1098-3015(17)30487-4/fulltext).

\section{Disclosure}

$\mathrm{MB}$ and MK are employees of Merck Healthcare KGaA, Darmstadt, Germany. YZ and HP are employees of EMD Serono, Rockland, MA (a business of Merck Healthcare $\mathrm{KGaA}$ ). The authors report no other conflicts of interest in this work.

\section{References}

1. Desch L, Kunstfeld R. Merkel cell carcinoma: chemotherapy and emerging new therapeutic options. J Skin Cancer. 2013;2013:327150. doi:10.1155/2013/327150

2. Ma JE, Brewer JD. Merkel cell carcinoma in immunosuppressed patients. Cancers (Basel). 2014;6(3):1328-1350. doi:10.3390/ cancers6031328

3. Feng H, Shuda M, Chang Y, et al. Clonal integration of a polyomavirus in human Merkel cell carcinoma. Science. 2008;319 (5866):1096-1100. doi:10.1126/science.1152586

4. Paulson KG, Park SY, Vandeven NA, et al. Merkel cell carcinoma: current US incidence and projected increases based on changing demographics. J Am Acad Dermatol. 2018;78(3):457-463. doi:10.1016/j.jaad.2017.10.028

5. Agelli M, Clegg LX. Epidemiology of primary Merkel cell carcinoma in the United States. J Am Acad Dermatol. 2003;49(5):832-841. doi:10.1067/S0190 
6. Grabowski J, Saltzstein SL, Sadler GR, Tahir Z, Blair S. A comparison of merkel cell carcinoma and melanoma: results from the California cancer registry. Clin Med Oncol. 2008;2:327-333.

7. Iyer JG, Parvathaneni U, Gooley T, et al. Single-fraction radiation therapy in patients with metastatic Merkel cell carcinoma. Cancer Med. 2015;4(8):1161-1170. doi:10.1002/cam4.458

8. Miller NJ, Bhatia S, Parvathaneni U, Iyer JG, Nghiem P. Emerging and mechanism-based therapies for recurrent or metastatic Merkel cell carcinoma. Curr Treat Options Oncol. 2013;14(2):249-263. doi:10.1007/s11864-013-0225-9

9. Hughes MP, Hardee ME, Cornelius LA, Hutchins LF, Becker JC, Gao L. Merkel cell carcinoma: epidemiology, target, and therapy. Curr Dermatol Rep. 2014;3:46-53. doi:10.1007/s13671-014-0068-z

10. Garibyan L, Cotter SE, Hansen JL, et al. Palliative treatment for intransit cutaneous metastases of Merkel cell carcinoma using surfacemold computer-optimized high-dose-rate brachytherapy. Cancer J. 2013;19(4):283-287. doi:10.1097/PPO.0b013e31829e3566

11. Lemos BD, Storer BE, Iyer JG, et al. Pathologic nodal evaluation improves prognostic accuracy in Merkel cell carcinoma: analysis of 5823 cases as the basis of the first consensus staging system. $J$ Am Acad Dermatol. 2010;63(5):751-761. doi:10.1016/j. jaad.2010.02.056

12. Santamaria-Barria JA, Boland GM, Yeap BY, et al. Merkel cell carcinoma: 30-year experience from a single institution. Ann Surg Oncol. 2013;20(4):1365-1373. doi:10.1245/s10434-012-2779-3

13. Becker JC, Lorenz E, Ugurel S, et al. Evaluation of real-world treatment outcomes in patients with distant metastatic Merkel cell carcinoma following second-line chemotherapy in Europe. Oncotarget. 2017;8 (45):79731-79741. doi:10.18632/oncotarget.19218

14. Cowey CL, Mahnke L, Espirito J, et al. Real-world outcomes of patients with metastatic merkel cell carcinoma treated with secondline or later chemotherapy in a community oncology setting in the United States. Future Oncol. 2017;13(19):1699-1710. doi:10.2217/ fon-2017-0187

15. NCCN Evidence Blocks ${ }^{\mathrm{TM}}$ for Merkel Cell Carcinoma V.2.2018. Referenced with permission from the NCCN Clinical Practice Guidelines in Oncology (NCCN Guidelines ${ }^{\circledR}$ ) with NCCN Evidence Blocks ${ }^{\mathrm{TM}}$ for Merkel Cell Carcinoma V.2.2018. (C) National Comprehensive Cancer Network, Inc.; 2018. All rights reserved. Available from: NCCN.org. Accessed October 29, 2018.

16. Lebbe C, Becker JC, Grob JJ, et al. Diagnosis and treatment of Merkel Cell Carcinoma. European consensus-based interdisciplinary guideline. Eur J Cancer. 2015;51(16):2396-2403. doi:10.1016/j. ejca.2015.06.131

17. Iyer JG, Blom A, Doumani R, et al. Response rate and durability of chemotherapy for metastatic Merkel cell carcinoma among 62 patients. J Clin Oncol. 2014;32:5s. doi:10.1200/ jco.2014.32.15_suppl.9091

18. Iyer JG, Blom A, Doumani R, et al. Response rates and durability of chemotherapy among 62 patients with metastatic Merkel cell carcinoma. Cancer Med. 2016;5(9):2294-2301. doi:10.1002/cam4.815

19. Satpute S, Ammakkanavar NR, Einhorn LH. Role of platinum-based chemotherapy for Merkel cell tumor in adjuvant and metastatic settings. J Clin Oncol. 2014;32:5s: Abstract No: 9049. doi:10.1200/ jco.2014.32.15_suppl.9049.

20. Sharma D, Flora G, Grunberg SM. Chemotherapy of metastatic Merkel cell carcinoma: case report and review of the literature. $\mathrm{Am}$ J Clin Oncol. 1991;14(2):166-169.

21. Tai PT, Yu E, Winquist E, et al. Chemotherapy in neuroendocrine/Merkel cell carcinoma of the skin: case series and review of 204 cases. J Clin Oncol. 2000;18(12):2493-2499. doi:10.1200/JCO.2000.18.12.2493

22. Voog E, Biron P, Martin JP, et al. Chemotherapy for patients with locally advanced or metastatic Merkel cell carcinoma. Cancer. 1999;85(12):2589-2595. doi:10.1002/(SICI)1097-0142(19990615) 85:12<2589::AID-CNCR15>3.0.CO;2-F
23. Bhatia S, Afanasiev O, Nghiem P. Immunobiology of Merkel cell carcinoma: implications for immunotherapy of a polyomavirus-associated cancer. Curr Oncol Rep. 2011;13(6):488-497. doi:10.1007/s11912-011$0197-5$

24. Gorayski P, Tripcony L, Poulsen M. Chemotherapy compliance in high-risk Merkel cell carcinoma treated with chemoradiotherapy. Australas J Dermatol. 2017;58(1):35-41. doi:10.1111/ajd.12419

25. Kearney M, Thokagevistk K, Boutmy E, et al. Treatment patterns, comorbidities, healthcare resource use, and associated costs by line of chemotherapy and level of comorbidity in patients with newlydiagnosed Merkel cell carcinoma in the United States. J Med Econ. 2018;21(12):1159-1171. doi:10.1080/13696998.2018.1517089

26. U.S. National Library of Medicine. Label: BAVENCIO - avelumab injection, solution, concentrate. DailyMed; October 2018. Available from: https://dailymed.nlm.nih.gov/dailymed/drugInfo.cfm?setid= 5cd725a1-2fa4-408a-a651-57a7b84b2118. Accessed December 2018.

27. U.S. National Library of Medicine. Label: KEYTRUDA- pembrolizumab injection, powder, lyophilized, for solution. DailyMed; November 2018. Avaialble from: https://dailymed.nlm.nih.gov/dai $1 \mathrm{ymed} / \mathrm{drug}$ Info.cfm? setid=9333 c 79b-d48 7-4538-a 9f0 71b91a02b287. Accessed December 2018.

28. U.S. National Library of Medicine. Label: OPDIVO- nivolumab injection. DailyMed; November 2018. Avaialble from: https://dai lymed.nlm.nih.gov/dailymed/drugInfo.cfm?setid=f570b9c4-68464de2-abfa-4d0a4ae4e394. Accessed December 2018.

29. Sullivan SD, Mauskopf JA, Augustovski F, et al. Budget impact analysis-principles of good practice: report of the ISPOR 2012 Budget Impact Analysis Good Practice II Task Force. Value Health. 2014;17(1):5-14. doi:10.1016/j.jval.2013.08.2291

30. Pannier A, Dunn JD. AMCP format for formulary submissions, Version 4.0. J Manag Care Spec Pharm. 2016;22(5):448.

31. United States Department of Labor. Medical CPI. Bureau of Labor Statistics; 2018. Avaialble from: https://www.bls.gov. Accessed August 2018.

32. D'Angelo S, Russell J, Lebbe C, et al. Efficacy and safety of first-line avelumab treatment in patients with stage IV metastatic merkel cell carcinoma - a preplanned interim analysis of a clinical trial. JAMA Oncol. 2018;4(9):e180077. doi:10.1001/jamaoncol.2017.4473

33. Nghiem PT, Bhatia S, Lipson EJ, et al. Durable tumor regression and overall survival (OS) in patients with advanced Merkel cell carcinoma (aMCC) receiving pembrolizumab as first-line therapy. J Clin Oncol. 2019;37(9):693-702. doi:10.1200/JCO.18.01896

34. Kaufman HL, Russell J, Hamid O, et al. Updated efficacy of avelumab in patients with previously treated metastatic Merkel cell carcinoma after $\geq 1$ year of follow-up: JAVELIN Merkel 200, a phase 2 clinical trial. J Immunother Cancer. 2018;6(1):7. doi:10.1186/ s40425-017-0310-x

35. Bullement A, Nathan P, Willis A, et al. Cost-effectiveness of avelumab for metastatic merkel cell Carcinoma. Pharmacoecon Open. 2019. doi:10.1007/s41669-018-0115-y

36. Centers for Medicare \& Medicaid Services. 2018 ASP drug pricing files. Centers for Medicare \& Medicaid Services; 2018. https://www.cms.gov/ Medicare/Medicare-Fee-for-Service-Part-B-Drugs/ McrPartBDrugAvgSalesPrice/2018ASPFiles.html. Accessed September $8,2018$.

37. Chang A, Hunt M, Parkinson DR, Hochster H, Smith TJ. Phase II trial of carboplatin in patients with metastatic malignant melanoma. A report from the Eastern Cooperative Oncology Group. Am J Clin Oncol. 1993;16(2):152-155.

38. Socinski MA, Smit EF, Lorigan P, et al. Phase III study of pemetrexed plus carboplatin compared with etoposide plus carboplatin in chemotherapy-naive patients with extensive-stage small-cell lung cancer. J Clin Oncol. 2009;27(28):4787-4792. doi:10.1200/ JCO.2009.23.1548 
39. Sun Y, Cheng Y, Hao X, et al. Randomized phase III trial of amrubicin/cisplatin versus etoposide/cisplatin as first-line treatment for extensive small-cell lung cancer. BMC Cancer. 2016;16:265. doi:10.1186/s12885-016-2301-6

40. Gervais R, Le Caer H, Monnet I, et al. Second-line oral chemotherapy (lomustine, cyclophosphamide, etoposide) versus intravenous therapy (cyclophosphamide, doxorubicin, and vincristine) in patients with relapsed small cell lung cancer: a randomized phase II study of GFPC 0501. Clin Lung Cancer. 2015;16(2):100-105. doi:10.1016/j. cllc.2014.10.002

41. Von Pawel J, Gatzemeier U, Pujol JL, et al. Phase II comparator study of oral versus intravenous topotecan in patients with chemosensitive small-cell lung cancer. J Clin Oncol. 2001;19(6):17431749. doi:10.1200/JCO.2001.19.6.1743

42. Wong W, Yim YM, Cloutier M, et al. Assessment of costs associated with adverse events in patients with cancer. PLoS One. 2018;13(4): e0196007. doi:10.1371/journal.pone.0196007

43. Wang PF, Chen Y, Song SY, et al. Immune-related adverse events associated with anti-PD-1/PD-L1 treatment for malignancies: a metaanalysis. Front Pharmacol. 2017;8:730. doi:10.3389/ fphar.2017.00730

44. US Department of Health \& Human Services. Healthcare cost and utilization project. Agency for Healthcare Research and Quality. Avaialble from: https:/hcupnet.ahrq.gov/\#setup. Accessed September 28, 2018.

45. Findacode.com. CPT codes. Findacode.com. Avaialble from: https:// www.findacode.com/. Accessed September 28, 2018.
46. Dimitroulis J, Rapti A, Stathopoulos GP, et al. Comparison of cisplatinpaclitaxel combination versus cisplatin-etoposide in patients with smallcell lung cancer: A Phase III study. Oncol Rep. 2008;20(4):879-884.

47. Loveman E, Jones J, Hartwell D, et al. The clinical and a cost effectiveness of topotecan for small cell lung cancer: a systematic review and economic evaluation. Health Technol Assess (Rockv). 2010;14:19. doi:10.3310/hta14410

48. Centers for Medicare \& Medicaid Services. Physician fee schedule. Centers for Medicare \& Medicaid Services; 2018. Avaialble from https:/www.cms.gov/apps/physician-fee-schedule/. Accessed September 8, 2018.

49. Centers for Medicare \& Medicaid Services. Clinical laboratory fee schedule. Centers for Medicare \& Medicaid Services; 2018. Available from: https://www.cms.gov/Medicare/Medicare-Fee-for-Service-Payment/ ClinicalLabFeeSched/index.html. Accessed September 8, 2018.

50. Steuten L, Fedorenko C, Sun Q, et al. Treatment patterns and predictors for overall survival in patients with metastatic Merkel cell carcinoma in the United States. Podium presented at the ISPOR 22nd Annual International Meeting; May 20-24, 2017; Boston, MA.

51. National population projections: United States by age, gender, ethnicity and race for years 2014-2060, released by the U.S. Census Bureau on December 10, 2014, on CDC WONDER Online Database; 2015. Avaialble from: http://wonder.cdc.gov/populationprojections-2014-2060.html. Accessed August 8, 2018.

52. Nghiem PT, Bhatia S, Lipson EJ, et al. PD-1 blockade with pembrolizumab in advance merkel-cell carcinoma. $N$ Engl J Med. 2016;374 (26):2542-2552. doi:10.1056/NEJMoa1603702
ClinicoEconomics and Outcomes Research

\section{Publish your work in this journal}

ClinicoEconomics and Outcomes Research is an international, peerreviewed open-access journal focusing on Health Technology Assessment, Pharmacoeconomics and Outcomes Research in the areas of diagnosis, medical devices, and clinical, surgical and pharmacological intervention. The economic impact of health policy and health systems organization also constitute important areas of coverage. The manuscript management system is completely online and includes a very quick and fair peer-review system, which is all easy to use. Visit http://www.dovepress.com/testimonials.php to read real quotes from published authors. 